\title{
Monotone-Iterative Method for the Initial Value Problem with Initial Time Difference for Differential Equations with "Maxima"
}

\author{
S. Hristova and A. Golev \\ Faculty of Mathematics and Informatics, Plovdiv University, Tzar Asen 24, 4000 Plovdiv, Bulgaria \\ Correspondence should be addressed to S. Hristova, snehri@gmail.com
}

Received 13 February 2012; Accepted 6 March 2012

Academic Editor: Josef Diblík

Copyright (C) 2012 S. Hristova and A. Golev. This is an open access article distributed under the Creative Commons Attribution License, which permits unrestricted use, distribution, and reproduction in any medium, provided the original work is properly cited.

The object of investigation of the paper is a special type of functional differential equations containing the maximum value of the unknown function over a past time interval. An improved algorithm of the monotone-iterative technique is suggested to nonlinear differential equations with "maxima." The case when upper and lower solutions of the given problem are known at different initial time is studied. Additionally, all initial value problems for successive approximations have both initial time and initial functions different. It allows us to construct sequences of successive approximations as well as sequences of initial functions, which are convergent to the solution and to the initial function of the given initial value problem, respectively. The suggested algorithm is realized as a computer program, and it is applied to several examples, illustrating the advantages of the suggested scheme.

\section{Introduction}

Equations with "maxima" find wide applications in the theory of automatic regulation. As a simple example of mathematical simulations by means of such equations, we shall consider the system of regulation of the voltage of a generator of constant current ([1]). The object of regulation is a generator of constant current with parallel stimulation, and the quantity regulated is the voltage on the clamps of the generator feeding an electric circuit with different loads. An equation with maxima is used if the regulator is constructed such that the maximal deviation of the quantity is regulated on the segment $[t-r, t]$. The equation describing the work of the regulator has the form

$$
T u^{\prime}(t)+u(t)+q \max _{s \in[t-r, t]} u(s)=f(t)
$$


where $T$ and $q$ are constant characterizing the object, $u(t)$ is the voltage regulated, and $f(t)$ is the perturbing effect.

Note that the above given model as well as all types of differential equations with "maxima" could be considered as delay functional differential equations. Differential equations with delay are studied by many authors (see, e.g., [2-14]). At the same time, the presence of maximum function in the equations causes impossibilities of direct application of almost all known results for delay functional differential equations (see the monograph [15]). Also, in the most cases, differential equations with "maxima," including the some linear scalar equations, are not possible to be solved in an explicit form. That requires the application of approximate methods. In the recent years, several effective approximate methods, based on the upper and lower solutions of the given problem, was proved for various problems of differential equations in [16-21].

In the current paper, an approximate method for solving the initial value problem for nonlinear differential equations with "maxima" is considered. This method is based on the method of lower and upper solutions. Meanwhile, in studying the initial value problems the authors usually keep the initial time unchanged. But, in the real repeated experiments it is difficult to keep this time fixed because of all kinds of disturbed factors. It requires the changing of initial time to be taken into consideration. Note that several qualitative investigations of the solutions of ordinary differential equations with initial time difference are studied in [22-30]. Also some approximate methods for various types of differential equations with initial time difference are proved in [23, 26, 31-33].

In this paper, an improved algorithm of monotone-iterative techniques is suggested to nonlinear differential equations with "maxima." The case when upper and lower solutions of the given problem are known at different initial time is studied. The behavior of the lower/upper solutions of the initial value problems with different initial times is studied. Also, some other improvements in the suggested algorithm are given. The main one is connected with the initial functions. In the known results for functional differential equations, the initial functions in the corresponding linear problems for successive approximations are the same (see, e.g., [20, 34-42]). In our case, it causes troubles with obtaining the solutions, which are successive approximations. To avoid these difficulties, we consider linear problems with different initial functions at any step. It allows us to chose appropriate initial data. Additionally, in connection with the presence of the maximum function in the equation and computer application of the suggested algorithm, an appropriate computer program is realized and it is applied to some examples illustrating the advantages and the practical application of the considered algorithm.

\section{Preliminary Notes and Definitions}

Consider the following initial value problem for the nonlinear differential equation with "maxima" (IVP):

$$
\begin{gathered}
x^{\prime}=f\left(t, x(t), \max _{s \in[t-r, t]} x(s)\right) \text { for } t \in\left[t_{0}, \infty\right), \\
x(t)=\varphi\left(t-t_{0}\right) \quad \text { for } t \in\left[t_{0}-r, t_{0}\right],
\end{gathered}
$$

where $x \in \mathbb{R}, t_{0} \geq 0, f: \mathbb{R}_{+} \times \mathbb{R} \times \mathbb{R} \rightarrow \mathbb{R}, \varphi(t):[-r, 0] \rightarrow \mathbb{R}$, and $r>0$ is a fixed constant. 
In the paper, we will study the differential equation with "maxima" (2.1) with an initial condition at two different initial points. For this purpose, we consider the differential equation (2.1) with the initial condition

$$
x(t)=\varphi\left(t-\tau_{0}\right) \quad \text { for } t \in\left[\tau_{0}-r, \tau_{0}\right]
$$

where $\tau_{0} \geq 0$.

Assume there exist a solution $x\left(t ; t_{0}, \varphi\right)$ of the IVP (2.1), (2.2) defined on $\left[t_{0}-r, t_{0}+T\right]$, and there exists a solution $y\left(t ; \tau_{0}, \varphi\right)$ of the IVP (2.1), (2.3) defined on $\left[\tau_{0}-r, \tau_{0}+T\right]$, where $T>0$ is a given constant. In the paper, we will compare the behavior of both solutions.

Definition 2.1. The function $\alpha \in C\left(\left[t_{0}-r, t_{0}+T\right), \mathbb{R}\right) \cup C^{1}\left(\left[t_{0}, t_{0}+T\right), \mathbb{R}\right)$ is called a lower (upper) solution of the IVP (2.1), (2.2) for $t \geq t_{0}-h$ if the following inequalities are satisfied:

$$
\begin{gathered}
\alpha^{\prime}(t) \leq(\geq) f\left(t, \alpha(t), \max _{s \in[t-r, t]} \alpha(s)\right) \quad \text { for } t \in\left[t_{0}, t_{0}+T\right], \\
\alpha(t) \leq(\geq) \varphi\left(t-t_{0}\right) \quad \text { for } t \in\left[t_{0}-r, t_{0}\right] .
\end{gathered}
$$

Note that the function $\alpha(t)$ is a lower (upper) solution of the IVP (2.1), (2.3) for $t \in$ $\left[\tau_{0}-r, \tau_{0}+T\right]$ if the point $t_{0}$ in the inequalities (2.4) is replaced by $\tau_{0}$.

\section{Comparison Results}

Often in the real world applications, the lower and upper solutions of one and the same differential equation are obtained at different initial time intervals.

The following result is a comparison result for lower and upper solutions with initial conditions given on different initial time intervals.

Theorem 3.1. Let the following conditions be satisfied.

(1) Let $t_{0}, \tau_{0} \geq 0$ be fixed such that $\eta=\tau_{0}-t_{0}>0$.

(2) The function $\alpha \in C\left(\left[t_{0}-r, t_{0}+T\right], \mathbb{R}\right) \cup C^{1}\left(\left[t_{0}, t_{0}+T\right], \mathbb{R}\right)$ is a lower solution of the IVP (2.1), (2.2).

(3) The function $\beta \in C\left(\left[\tau_{0}-r, \tau_{0}+T\right], \mathbb{R}\right) \cup C^{1}\left(\left[\tau_{0}, \tau_{0}+T\right], \mathbb{R}\right)$ is an upper solution of the IVP (2.1), (2.3).

(4) The function $f(t, u, v): \mathbb{R}_{+} \times \mathbb{R} \times \mathbb{R} \rightarrow \mathbb{R}$ is nondecreasing in its both first and third argument $t$ for any $u \in \mathbb{R}$, and there exist positive constants $L_{1}, L_{2}$ such that for $t \in \mathbb{R}_{+}$and $u_{1} \geq u_{2}$ and $v_{1} \geq v_{2}$, the inequality

$$
f\left(t, u_{1}, v_{1}\right)-f\left(t, u_{2}, v_{2}\right) \leq L_{1}\left(u_{1}-u_{2}\right)+L_{2}\left(v_{1}-v_{2}\right)
$$

holds.

(5) The function $\varphi(t) \in C([-r, 0], \mathbb{R})$.

Then $\alpha(t) \leq \beta(t+\eta)$ for $t \in\left[t_{0}, t_{0}+T\right]$. 
Proof. Choose a positive number $M$ such that $M>L_{1}+L_{2}$ and a positive number $\epsilon$. Define a function $w(t)=\beta(t+\eta)+\epsilon e^{M t}$ for $t \in\left[t_{0}, t_{0}+T\right]$ and $w(t)=\beta(t+\eta)+\epsilon e^{M t_{0}}$ for $t \in\left[t_{0}-r, t_{0}\right)$. Then for $t \in\left[t_{0}, t_{0}+T\right]$, the following inequalities

$$
\begin{gathered}
w(t)>\beta(t+\eta), \\
\max _{s \in[t-r, t]} w(s)=\max _{s \in[t-r, t]}\left(\beta(s+\eta)+\epsilon e^{M s}\right) \geq \max _{s \in[t-r, t]}(\beta(s+\eta))=\max _{s \in[t+\eta-r, t+\eta]} \beta(s), \\
\max _{s \in[t-r, t]} w(s) \leq \max _{s \in[t-r, t]}(\beta(s+\eta))+\epsilon e^{M t}=\max _{s \in[t+\eta-r, t+\eta]} \beta(s)+\epsilon e^{M t}
\end{gathered}
$$

hold.

Therefore, we obtain

$$
\begin{aligned}
w^{\prime}(t) \geq & f\left(t+\eta, \beta(t+\eta), \max _{s \in[t+\eta-r, t+\eta]} \beta(s)\right)+\epsilon M e^{M t} \\
\geq & -L_{1}(w(t)-\beta(t+\eta))-L_{2}\left(\max _{s \in[t-r, t]} w(s)-\max _{s \in[t+\eta-r, t+\eta]} \beta(s)\right) \\
& +f\left(t+\eta, w(t), \max _{s \in[t-r, t]} w(s)\right)+\epsilon M e^{M t} \\
\geq & f\left(t+\eta, w(t), \max _{s \in[t-r, t]} w(s)\right)+\epsilon e^{M t}\left(M-L_{1}-L_{2}\right) \\
> & f\left(t+\eta, w(t), \max _{s \in[t-r, t]} w(s)\right) \geq f\left(t, w(t), \max _{s \in[t-r, t]} w(s)\right) .
\end{aligned}
$$

Note that for $t \in\left[t_{0}-r, t_{0}\right]$ we have $\alpha(t) \leq \varphi\left(t-t_{0}\right)=\varphi\left(t+\eta-\tau_{0}\right) \leq \beta(t+\eta)<w(t)$. We will prove that

$$
\alpha(t) \leq w(t) \quad \text { for } t \in\left[t_{0}-r, t_{0}+T\right]
$$

Assume the contrary, that is, there exists a point $t_{1}>t_{0}$ such that $\alpha(t)<w(t)$ for $t \in\left[t_{0}-r, t_{1}\right), \alpha\left(t_{1}\right)=w\left(t_{1}\right)$ and $\alpha(t) \geq w(t)$ for $t \in\left(t_{1}, t_{2}\right)$, where $t_{2}$ is sufficiently close to $t_{1}$. Therefore $\alpha^{\prime}\left(t_{1}\right) \geq w^{\prime}\left(t_{1}\right)$. Then $w^{\prime}\left(t_{1}\right) \leq \alpha^{\prime}\left(t_{1}\right) \leq f\left(t_{1}, \alpha\left(t_{1}\right), \max _{s \in\left[t_{1}-r, t_{1}\right]} \alpha(s)\right) \leq$ $f\left(t_{1}, w\left(t_{1}\right), \max _{s \in\left[t_{1}-r, t_{1}\right]} w(s)\right)<w^{\prime}\left(t_{1}\right)$. The obtained contradiction proves the claim.

Therefore, $\alpha(t) \leq w(t)<\beta(t+\eta)$ for $t \in\left[t_{0}-r, t_{0}+T\right]$.

Corollary 3.2. Let the conditions of Theorem 3.1 be fulfilled. Then $\alpha(t-\eta) \leq \beta(t)$ for $t \in\left[\tau_{0}-r, \tau_{0}+\right.$ $T]$.

The comparison results are true if the inequality $\tau_{0}<t_{0}$ holds.

Theorem 3.3 (comparison result). Let the following conditions be satisfied.

(1) The conditions 2, 3, 5 of Theorem 3.1 are satisfied.

(2) Let $t_{0}, \tau_{0} \geq 0$ be fixed such that $\eta=\tau_{0}-t_{0}<0$. 
(3) The function $f(t, u, v): \mathbb{R}_{+} \times \mathbb{R} \times \mathbb{R} \rightarrow \mathbb{R}$ is nonincreasing in its first argument and is nondecreasing in its third argument for any $u \in \mathbb{R}$, and there exist positive constants $L_{1}, L_{2}$ such that for $t \in \mathbb{R}_{+}$and $u_{1} \geq u_{2}$ and $v_{1} \geq v_{2}$ the inequality

$$
f\left(t, u_{1}, v_{1}\right)-f\left(t, u_{2}, v_{2}\right) \leq L_{1}\left(u_{1}-u_{2}\right)+L_{2}\left(v_{1}-v_{2}\right)
$$

holds.

Then $\alpha(t) \leq \beta(t+\eta)$ for $t \geq t_{0}$.

The proof of Theorem 3.3 is similar to the proof of Theorem 3.1 and we omit it.

In what follows, we shall need that the functions $\alpha, \beta \in C\left(\left[t_{0}-r, T+t_{0}\right], \mathbb{R}\right)$ are such that $\alpha(t) \leq \beta(t+\eta)$.

Consider the sets:

$$
\begin{aligned}
& S\left(\alpha, \beta, t_{0}, \eta\right)=\left\{u \in C\left(\left[t_{0}-r, T+t_{0}\right], \mathbb{R}\right): \alpha(t) \leq u(t) \leq \beta(t+\eta) \text { for } t \in\left[t_{0}-r, T+t_{0}\right]\right\}, \\
& \widetilde{S}\left(\alpha, \beta, \tau_{0}, \eta\right)=\left\{u \in C\left(\left[\tau_{0}-r, T+\tau_{0}\right], \mathbb{R}\right): \alpha(t-\eta) \leq u(t) \leq \beta(t) \text { for } t \in\left[\tau_{0}-r, T+\tau_{0}\right]\right\}, \\
& \Omega\left(\alpha, \beta, t_{0}\right)=\left\{(t, x, y) \in\left[t_{0}, T+t_{0}\right] \times \mathbb{R}^{2}: \alpha(t) \leq x \leq \beta(t), \max _{s \in[t-r, t]} \alpha(s) \leq y \leq \max _{s \in[t-r, t]} \beta(s)\right\} .
\end{aligned}
$$

In our further investigations, we will need the following comparison result on differential inequalities with "maxima."

Lemma 3.4 (see [43, Lemma 2.1]). Let the function $m \in C\left(\left[t_{0}-r, T+t_{0}\right], \mathbb{R}\right) \cup C^{1}\left(\left[t_{0}, T+t_{0}\right], \mathbb{R}\right)$ satisfies the inequalities

$$
\begin{gathered}
m^{\prime} \leq-L_{1} m(t)-L_{2} \min _{s \in[t-r, t]} m(s) \quad \text { for } t \in\left[t_{0}, T+t_{0}\right] \\
m(t) \leq 0 \text { for } t \in\left[t_{0}-r, t_{0}\right]
\end{gathered}
$$

where the positive constants $L_{1}, L_{2}$ are such that $\left(L_{1}+L_{2}\right) T \leq 1$.

Then the inequality $m(t) \leq 0$ holds on $\left[t_{0}-r, T+t_{0}\right]$.

In our further investigations, we will use the following result, which is a partial case of Theorem 3.1 [44].

Lemma 3.5 (existence and uniqueness result). Let the following conditions be fulfilled.

(1) The functions $Q \in C\left(\left[t_{0}, t_{0}+T\right], \mathbb{R}\right), q \in C\left(\left[t_{0}, t_{0}+T\right], \mathbb{R}\right)$.

(2) The functions $\varphi \in C([-r, 0], \mathbb{R})$.

Then the initial value problem for the linear scalar equation

$$
\begin{gathered}
u^{\prime}=L_{1} u(t)+L_{2} \max _{s \in[t-r, t]} u(s)+Q(t) \quad \text { for } t \in\left[t_{0}, T+t_{0}\right], \\
u(t)=\varphi\left(t-t_{0}\right) \quad \text { for } t \in\left[t_{0}-r, t_{0}\right]
\end{gathered}
$$

has a unique solution $u\left(t-t_{0}\right)$ on the interval $\left[t_{0}-r, T+t_{0}\right]$. 


\section{Main Results}

Denote $J_{1}=\left[t_{0}-r, T+t_{0}\right]$ and $J_{2}=\left[\tau_{0}-r, T+\tau_{0}\right]$, where $t_{0}, \tau_{0} \geq 0$ are fixed numbers.

Case 1. Let $t_{0} \leq \tau_{0}$.

Theorem 4.1. Let the following conditions be fulfilled.

(1) The points $t_{0}, \tau_{0} \geq 0$ are such that $\eta=\tau_{0}-t_{0} \geq 0$.

(2) The function $\alpha_{0}(t) \in C\left(\left[t_{0}-r, T+t_{0}\right], \mathbb{R}\right) \cup C^{1}\left(\left[t_{0}, T+t_{0}\right], \mathbb{R}\right)$ is a lower solution of the $\operatorname{IVP}(2.1),(2.2)$ in $J_{1}$.

(3) The function $\beta_{0}(t) \in C\left(\left[\tau_{0}-r, T+\tau_{0}\right], \mathbb{R}\right) \cup C^{1}\left(\left[\tau_{0}, T+\tau_{0}\right], \mathbb{R}\right)$ is an upper solution of the $\operatorname{IVP}(2.1),(2.3)$ in $J_{2}$.

(4) The function $\varphi \in C([-r, 0], \mathbb{R})$.

(5) The function $f \in C\left(\Omega\left(\alpha_{0}, \beta_{0}, t_{0}\right), \mathbb{R}\right)$ is nondecreasing in its first argument and satisfies the one side Lipschitz condition

$$
f\left(t, u_{1}, v_{1}\right)-f\left(t, u_{2}, v_{2}\right) \geq L_{1}\left(u_{2}-u_{1}\right)+L_{2}\left(v_{2}-v_{1}\right),
$$

for $u_{1} \leq u_{2}$ and $v_{1} \leq v_{2}$, where $L_{1}, L_{2}>0$ are such that

$$
\left(L_{1}+L_{2}\right) T \leq 1
$$

Then there exist two sequences of functions $\left\{\alpha_{n}(t)\right\}_{0}^{\infty}$ and $\left\{\beta_{n}(t)\right\}_{0}^{\infty}$ such that

(a) the functions $\alpha_{n}(t)=1,2, \ldots$ are lower solutions of the IVP (2.1), (2.2) on $\left[t_{0}-r, T+t_{0}\right]$;

(b) the functions $\beta_{n}(t)=1,2, \ldots$ are upper solutions of the IVP (2.1), (2.3) on $\left[\tau_{0}-r, T+\tau_{0}\right]$;

(c) the sequence $\left\{\alpha_{n}(t)\right\}_{0}^{\infty}$ is increasing;

(d) the sequence $\left\{\beta_{n}(t)\right\}_{0}^{\infty}$ is decreasing;

(e) the inequalities

$$
\begin{array}{ll}
\alpha_{0}(t) \leq \cdots \leq \alpha_{n}(t) \leq \beta_{n}(t+\eta) \leq \cdots \leq \beta_{0}(t+\eta), & t \in\left[t_{0}-r, T+t_{0}\right], \\
\alpha_{0}(t-\eta) \leq \cdots \leq \alpha_{n}(t-\eta) \leq \beta_{n}(t) \leq \cdots \leq \beta_{0}(t), & t \in\left[\tau_{0}-r, T+\tau_{0}\right]
\end{array}
$$

hold;

(f) both sequences uniformly converge and $x(t)=\lim _{n \rightarrow \infty} \alpha_{n}(t)$ is a solution of the IVP (2.1), (2.2) in $S\left(\alpha_{0}, \beta_{0}, t_{0}, \eta\right)$, and $y(t)=\lim _{n \rightarrow \infty} \beta_{n}(t)$ is a solution of the IVP (2.1), (2.3) in $\widetilde{S}\left(\alpha_{0}, \beta_{0}, \tau_{0}, \eta\right)$.

Proof. According to Theorem 3.1, the inequality $\alpha_{0}(t) \leq \beta_{0}(t+\eta)$ holds on $\left[t_{0}-r, T+t_{0}\right]$. Let $L_{0}=\min _{s \in\left[t_{0}-r, t_{0}\right]}\left(\varphi\left(s-t_{0}\right)-\alpha_{0}(s)\right) \geq 0$. Choose a number $k_{0} \in[0,1)$ such that

$$
k_{0} \leq L_{0} .
$$


Therefore, $\varphi\left(t-t_{0}\right)-\alpha_{0}(t) \geq L_{0}>k_{0} L_{0}$ or $\alpha_{0}(t) \leq \varphi\left(t-t_{0}\right)-k_{0} L_{0}$ on $\left[t_{0}-r, t_{0}\right]$. We consider the linear differential equation with "maxima"

$$
\begin{aligned}
x^{\prime}(t)= & f\left(t, \alpha_{0}(t), \max _{s \in[t-r, t]} \alpha_{0}(s)\right)-L_{1}\left(x(t)-\alpha_{0}(t)\right) \\
& -L_{2}\left(\max _{s \in[t-r, t]} x(s)-\max _{s \in[t-r, t]} \alpha_{0}(s)\right) \text { for } t \in\left[t_{0}, T+t_{0}\right]
\end{aligned}
$$

with the initial condition

$$
x(t)=\varphi\left(t-t_{0}\right)-k_{0} L_{0} \quad \text { for } t \in\left[t_{0}-r, t_{0}\right] .
$$

According to Lemma 3.5, the linear initial value problem (4.5), (4.6) has a unique solution $\alpha_{1}(t)$ in $J_{1}$.

We will prove that $\alpha_{0}(t) \leq \alpha_{1}(t)$ on $J_{1}$.

From the choice of $L_{0}, k_{0}$ and equality (4.6), it follows that $\alpha_{0}(t) \leq \varphi\left(t-t_{0}\right)-k_{0} L_{0}=\alpha_{1}(t)$ for $t \in\left[t_{0}-r, t_{0}\right]$.

Now, let $t \in\left[t_{0}, T+t_{0}\right]$. Consider the function $u(t)=\alpha_{0}(t)-\alpha_{1}(t)$ defined on $J_{1}$. It is clear that $u(t) \leq 0$ on $\left[t_{0}-r, t_{0}\right]$. From the definition of the function $\alpha_{0}(t)$ and (4.5), we have

$$
u^{\prime}(t) \leq-L_{1} u(t)-L_{2}\left(\max _{s \in[t-r, t]} \alpha_{0}(s)-\max _{s \in[t-r, t]} \alpha_{1}(s)\right) \text { for } t \in\left[t_{0}, T+t_{0}\right]
$$

Now from inequality (4.7) and

$$
\begin{aligned}
\max _{s \in[t-h, t]} \alpha_{0}(s)-\max _{s \in[t-r, t]} \alpha_{1}(s) & =\max _{s \in[t-r, t]} \alpha_{0}(s)-\alpha_{1}(\xi) \\
& \geq \alpha_{0}(\xi)-\alpha_{1}(\xi) \\
& \geq \min _{s \in[t-r, t]}\left(\alpha_{0}(s)-\alpha_{1}(s)\right) \\
& =\min _{s \in[t-r, t]} u(s)
\end{aligned}
$$

we obtain

$$
u^{\prime}(t) \leq-L_{1} u(t)-L_{2} \min _{s \in[t-r, t]} u(s), \quad t \in\left[t_{0}, T+t_{0}\right]
$$

According to Lemma 3.4, the inequality $u(t) \leq 0$ holds on $J_{1}$, that is, $\alpha_{0}(t) \leq \alpha_{1}(t)$.

We will prove that the function $\alpha_{1}(t)$ is a lower solution of the IVP $(2.1),(2.2)$ on $J_{1}$.

From equality (4.6), it follows the validity of the inequality $\alpha_{1}(t) \leq \varphi\left(t-t_{0}\right)$ on $\left[t_{0}-r, t_{0}\right]$. Now, let $t \in\left[t_{0}, T+t_{0}\right]$. Then, since $\alpha_{0}(t) \leq \alpha_{1}(t)$, according to the one side Lipschitz condition 2 of Theorem 4.1, we have

$$
\begin{aligned}
\alpha_{1}^{\prime}(t) & =Q(t)-L_{1}\left(\alpha_{1}(t)-\alpha_{0}(t)\right)-L_{2}\left(\max _{s \in[t-r, t]} \alpha_{1}(s)-\max _{s \in[t-r, t]} \alpha_{0}(s)\right) \\
& =f\left(t, \alpha_{1}(t), \max _{s \in[t-r, t]} \alpha_{1}(s)\right)+f\left(t, \alpha_{0}(t), \max _{s \in[t-r, t]} \alpha_{0}(s)\right)
\end{aligned}
$$




$$
\begin{aligned}
& -f\left(t, \alpha_{1}(t), \max _{s \in[t-r, t]} \alpha_{1}(s)\right)-L_{1}\left(\alpha_{1}(t)-\alpha_{0}(t)\right) \\
& -L_{2}\left(\max _{s \in[t-r, t]} \alpha_{1}(s)-\max _{s \in[t-r, t]} \alpha_{0}(s)\right) \\
\leq & f\left(t, \alpha_{1}(t), \max _{s \in[t-r, t]} \alpha_{1}(s)\right) \text { for } t \in\left[t_{0}, T+t_{0}\right],
\end{aligned}
$$

where $Q(t)=f\left(t, \alpha_{0}(t), \max _{s \in[t-r, t]} \alpha_{0}(s)\right)$.

Next, according to Lemma 3.4 for the function $u(t)=\alpha_{1}(t)-\beta_{0}(t-\eta)$, the inequality $\alpha_{1}(t) \leq \beta_{0}(t+\eta)$ holds on $J_{1}$, that is, the inclusion $\alpha_{1} \in S\left(\alpha_{0}, \beta_{0}, t_{0}, \eta\right)$ is valid.

Let $C_{0}=\min _{s \in\left[\tau_{0}-r, \tau_{0}\right]}\left(\beta_{0}(s)-\varphi\left(s-\tau_{0}\right)\right) \geq 0$. Choose a number $p_{0} \in[0,1)$ such that

$$
p_{0} \leq C_{0}
$$

Therefore, $\beta_{0}(t)-\varphi\left(t-\tau_{0}\right) \geq C_{0}>p_{0} C_{0}$ or $\beta_{0}(t) \geq \varphi\left(t-\tau_{0}\right)+p_{0} C_{0}$ on $\left[\tau_{0}-r, \tau_{0}\right]$.

We consider the linear differential equation with "maxima"

$$
\begin{aligned}
x^{\prime}(t)= & f\left(t, \beta_{0}(t), \max _{s \in[t-r, t]} \beta_{0}(s)\right)-L_{1}\left(x(t)-\beta_{0}(t)\right) \\
& -L_{2}\left(\max _{s \in[t-r, t]} x(s)-\max _{s \in[t-r, t]} \beta_{0}(s)\right) \text { for } t \in\left[\tau_{0}, T+\tau_{0}\right]
\end{aligned}
$$

with the initial condition

$$
x(t)=\varphi\left(t+\tau_{0}\right)+p_{0} C_{0} \quad \text { for } t \in\left[\tau_{0}-r, \tau_{0}\right] .
$$

There exists a unique solution $\beta_{1}(t)$ of the IVP (4.12), (4.13), which is defined on $J_{2}$.

The function $\beta_{1}(t)$ is an upper solution of the IVP (2.1), (2.3) on $J_{2}$, and the inclusion $\beta_{1} \in \widetilde{S}\left(\alpha_{0}, \beta_{0}, \tau_{0}, \eta\right)$ is valid. The proofs are similar to the ones about the function $\alpha_{1}$. We omit the proofs.

Functions $\alpha_{1}(t), \beta_{1}(t)$ are a lower and an upper solution of the IVP (2.1), (2.2) and (2.1), (2.3) correspondingly. According to Lemma 3.4 the inequality $\alpha_{1}(t) \leq \beta_{1}(t+\eta)$ on $J_{1}$ holds.

Similarly, recursively, we can construct two sequences of functions $\left\{\alpha_{n}(t)\right\}_{0}^{\infty}$ and $\left\{\beta_{n}(t)\right\}_{0}^{\infty}$. In fact, if the functions $\alpha_{n}(t)$ and $\beta_{n}(t)$ are known, and $L_{n}=\min _{s \in[-r, 0]}(\varphi(s)-$ $\left.\alpha_{n}(s)\right), C_{n}=\min _{s \in[-r, 0]}\left(\beta_{n}(s)-\varphi(s)\right)$, and the numbers $k_{n}, p_{n} \in[0,1)$ are such that

$$
k_{n} \leq L_{n}, \quad p_{n} \leq C_{n},
$$


then the function $\alpha_{n+1}(t)$ is the unique solution of the initial value problem for the linear differential equation with "maxima"

$$
\begin{aligned}
x^{\prime}= & f\left(t, \alpha_{n}(t), \max _{s \in[t-r, t]} \alpha_{n}(s)\right)-L_{1}\left(x-\alpha_{n}(t)\right) \\
& -L_{2}\left(\max _{s \in[t-r, t]} x(s)-\max _{s \in[t-r, t]} \alpha_{n}(s)\right) \text { for } t \in\left[t_{0}, T+t_{0}\right], \\
x(t)= & \varphi(t)-k_{n} L_{n} \quad \text { for } t \in\left[t_{0}-r, t_{0}\right],
\end{aligned}
$$

and the function $\beta_{n+1}(t)$ is the unique solution of the initial value problem

$$
\begin{aligned}
x^{\prime}(t)= & f\left(t, \beta_{n}(t), \max _{s \in[t-r, t]} \beta_{n}(s)\right)-L_{1}\left(x-\beta_{n}(t)\right) \\
& -L_{2}\left(\max _{s \in[t-h, t]} x(s)-\max _{s \in[t-r, t]} \beta_{n}(s)\right) \text { for } t \in\left[\tau_{0}, T+\tau_{0}\right], \\
x(t)= & \varphi(t)+p_{n} C_{n} \quad \text { for } t \in\left[\tau_{0}-r, \tau_{0}\right] .
\end{aligned}
$$

Now following exactly as for the case $n=0$, it can be proved that the function $\alpha_{n+1}(t)$ is a lower solution of the IVP (2.1), (2.2) on $J_{1}$, the function $\beta_{n+1}(t)$ is an upper solution of the IVP (2.1), (2.3) on $J_{2}$, the inclusions $\alpha_{n+1} \in S\left(\alpha_{n}, \beta_{n}, t_{0}, \eta\right), \beta_{n+1} \in \widetilde{S}\left(\alpha_{n}, \beta_{n}, \tau_{0}, \eta\right)$ are valid, and the inequalities (4.3) hold.

Therefore, the sequence $\left\{\alpha_{n}(t)\right\}_{0}^{\infty}$ is uniformly convergent on $J_{1}$ and $\left\{\beta_{n}(t)\right\}_{0}^{\infty}$ is uniformly convergent on $J_{2}$.

Denote

$$
\begin{array}{ll}
\lim _{n \rightarrow \infty} \alpha_{n}(t)=u(t), & t \in\left[t_{0}-r, T+t_{0}\right], \\
\lim _{n \rightarrow \infty} \beta_{n}(t)=v(t), & t \in\left[\tau_{0}-r, T+\tau_{0}\right] .
\end{array}
$$

From the uniform convergence and the definition of the functions $\alpha_{n}(t)$ and $\beta_{n}(t)$, it follows that the following inequalities hold

$$
\alpha_{0}(t) \leq u(t) \leq v(t+\eta) \leq \beta_{0}(t+\eta), \quad t \in\left[t_{0}-r, T+t_{0}\right]
$$

Case 2. Let $t_{0}>\tau_{0}$.

In this case, we could approximate again the solution of the given initial value problem, starting from lower and upper solutions given at two different initial points. Since the proofs are similar, we will set up only the results.

Theorem 4.2. Let the following conditions be fulfilled.

(1) The points $t_{0}, \tau_{0} \geq 0$ are such that $\eta=t_{0}-\tau_{0} \geq 0$.

(2) The conditions 2, 3, 4 of Theorem 4.1 are satisfied. 
(3) The function $f \in C\left(\Omega\left(\alpha_{0}, \beta_{0}, \tau_{0}\right), \mathbb{R}\right)$ is nonincreasing in its first argument and satisfies the one side Lipschitz condition

$$
f\left(t, u_{1}, v_{1}\right)-f\left(t, u_{2}, v_{2}\right) \geq L_{1}\left(u_{2}-u_{1}\right)+L_{2}\left(v_{2}-v_{1}\right)
$$

for $u_{1} \leq u_{2}$ and $v_{1} \leq v_{2}$, where $L_{1}, L_{2}>0$ are such that

$$
\left(L_{1}+L_{2}\right) T \leq 1
$$

Then there exist two sequences of functions $\left\{\alpha_{n}(t)\right\}_{0}^{\infty}$ and $\left\{\beta_{n}(t)\right\}_{0}^{\infty}$ such that

(a) the functions $\alpha_{n}(t)=1,2, \ldots$ are lower solutions of the IVP (2.1), (2.2) on $\left[t_{0}-r, T+t_{0}\right]$;

(b) the functions $\beta_{n}(t)=1,2, \ldots$ are upper solutions of the IVP (2.1), (2.3) on $\left[\tau_{0}-r, T+\tau_{0}\right]$;

(c) the sequence $\left\{\alpha_{n}(t)\right\}_{0}^{\infty}$ is increasing;

(d) the sequence $\left\{\beta_{n}(t)\right\}_{0}^{\infty}$ is decreasing;

(e) the inequalities

$$
\begin{array}{ll}
\alpha_{0}(t) \leq \cdots \leq \alpha_{n}(t) \leq \beta_{n}(t+\eta) \leq \cdots \leq \beta_{0}(t+\eta), & t \in\left[t_{0}-r, T+t_{0}\right], \\
\alpha_{0}(t-\eta) \leq \cdots \leq \alpha_{n}(t-\eta) \leq \beta_{n}(t) \leq \cdots \leq \beta_{0}(t), & t \in\left[\tau_{0}-r, T+\tau_{0}\right]
\end{array}
$$

hold;

(f) both sequences uniformly converge and $x(t)=\lim _{n \rightarrow \infty} \alpha_{n}(t)$ is a solution of the IVP (2.1), (2.2) in $S\left(\alpha_{0}, \beta_{0}, t_{0}, \eta\right)$ and $y(t)=\lim _{n \rightarrow \infty} \beta_{n}(t)$ is a solution of the IVP (2.1), (2.3) in $\widetilde{S}\left(\alpha_{0}, \beta_{0}, \tau_{0}, \eta\right)$.

\section{Computer Realization}

Since the set of differential equations, which could be solved in an explicit form, is very narrow, we will realize the above suggested algorithm numerically. At present, we cannot solve numerically differential equations with "maxima" by the existing ready-made systems, such as Mathematica, Mathlab, and so forth, because of the maximum of the unknown function over a past time interval. It requires proposed and computer realized new algorithms for solving such kind of equations. In [43], an algorithm for solving a class of differential equations with "maxima," based on the trapezoid's method, is presented. The main problem in the suggested algorithm is obtaining the maximum of the unknown function over a past time interval. It is based on the idea that the local maximum depends on values that are initially inserted and later they are removed within the range. A special structure is applied to help quicker obtaining the local maximum ([43]). The suggested scheme could be based on any numerical method for solving differential equations. In this paper, we will use Euler method in the application of the algorithm ([43]) for solving differential equations with "maxima." We combine the algorithm of Euler method and obtaining of maximum of the function, and we get the following modification:

$$
\begin{gathered}
t_{k+1}=t_{k}+h, \\
y_{n+1}\left(t_{k+1}\right)=y_{n+1}\left(t_{k}\right)+h f\left(t_{k}, y_{n+1}\left(t_{k}\right), y_{n}\left(t_{k}\right), \max y_{n, k}, \max y_{n+1, k}\right),
\end{gathered}
$$


where the values $\max y_{n, k}=\max _{s \in\left[t_{k}-r, t_{k}\right]} y_{n}(s)$ and $\max y_{n+1, k}=\max _{s \in\left[t_{k}-r, t_{k}\right]} y_{n+1}(s)$ are obtained by the algorithm suggested in [43], and the initial values of $y_{n+1}\left(t_{0}\right)$ are found by initial conditions (4.16) and (4.18).

\section{Applications}

Now we will illustrate the employment of the suggested above scheme to a particular nonlinear scalar differential equations with "maxima."

Initially we will consider the case of one and the same initial points of both initial value problems. By this way, we will emphasize our considerations to the advantages of the involved in the initial conditions constants.

Example 6.1. Consider the following scalar nonlinear differential equation with "maxima":

$$
x^{\prime}=\frac{1}{1-x(t)}-2 \max _{s \in[t-0.1, t]} x(s)-1, \quad \text { for } t \in[0,0.35]
$$

with the initial condition

$$
x(t)=0, \quad t \in[-0.1,0],
$$

where $x \in \mathbb{R}$. It is easy to check that the initial value problem (6.1), (6.2) has a zero solution.

In this case $f(t, x, y) \equiv 1 /(1-x)-2 y-1, t_{0}=\tau_{0}=0, T=0.35, r=0.1, J_{1}=J_{2}=$ $[-0.1,0.35]$. Choose $\alpha_{0}(t)=-1 / 4$ and $\beta_{0}(t)=1 / 4$. Then $\alpha_{0}(t)$ is a lower solution and $\beta_{0}(t)$ is an upper solution of the IVP (6.1), (6.2). In this case, $\Omega\left(\alpha_{0}, \beta_{0}, t_{0}\right)=\left\{(t, u, v) \in[0,0.35] \times \mathbb{R}^{2}\right.$ : $-1 / 4 \leq u, v \leq 1 / 4\}$. Let $\left(t, u_{1}, v_{1}\right),\left(t, u_{2}, v_{2}\right) \in \Omega\left(\alpha_{0}, \beta_{0}, t_{0}\right)$ and $u_{1} \leq u_{2}$ and $v_{1} \leq v_{2}$. Then

$$
\begin{aligned}
f\left(t, u_{1}, v_{1}\right)-f\left(t, u_{2}, v_{2}\right) & =\frac{1}{1-u_{1}}-\frac{1}{1-u_{2}}+2\left(v_{2}-v_{1}\right) \\
& =\frac{1}{\left(1-u_{1}\right)\left(1-u_{2}\right)}\left(u_{2}-u_{1}\right)+2\left(v_{2}-v_{1}\right) \\
& \geq \frac{16}{25}\left(u_{2}-u_{1}\right)+2\left(v_{2}-v_{1}\right) .
\end{aligned}
$$

That is, $L_{1}=0.64, L_{2}=2$, and the inequality $\left(L_{1}+L_{2}\right) T<1$ holds.

The successive approximations $\alpha_{n+1}(t)$ and $\beta_{n+1}(t)$ will be the unique solutions of the linear problems

$$
\begin{gathered}
x^{\prime}=\frac{1}{1-\alpha_{n}(t)}+0.64 \alpha_{n}(t)-1-0.64 x-2 \max _{s \in[t-0.1, t]} x(s) \text { for } t \in[0,0.35], \\
x(t)=-k_{n} L_{n} \quad \text { for } t \in[-0.1,0], \\
x^{\prime}(t)=\frac{1}{1-\beta_{n}(t)}-1+0.64 \beta_{n}(t)-0.64 x-2 \max _{s \in[t-0.1, t]} x(s) \text { for } t \in[0,0.35], \\
x(t)=p_{n} C_{n} \text { for } t \in[-0.1,0],
\end{gathered}
$$


where $L_{n}=\min _{s \in[-0.1,0]}\left(-\alpha_{n}(s)\right) \geq 0, C_{n}=\min _{s \in[-0.1,0]}\left(\beta_{n}(s)\right) \geq 0$, and the numbers $k_{n}, p_{n} \in$ $[0,1)$ are chosen such that $k_{n} \leq L_{n}, p_{n} \leq C_{n}, n=1,2,3, \ldots$.

Now we will construct an increasing sequence of lower solutions and a decreasing sequence of upper solutions, which will be convergent to the zero solution.

The first lower approximation $\alpha_{1}(t)$ is a solution of the IVP

$$
\begin{gathered}
x^{\prime}=-\frac{9}{25}-0.64 x-2 \max _{s \in[t-0.1, t]} x(s) \text { for } t \in[0,0.35], \\
x(t)=-\frac{k_{0}}{4}, \quad t \in[-0.1,0] .
\end{gathered}
$$

Choose $k_{0}=6 / 11$. Then the IVP (6.5) has an exact solution $\alpha_{1}(t)=-3 / 22$.

The second lower approximation $\alpha_{2}(t)$ is a solution of the IVP

$$
\begin{gathered}
x^{\prime}=-\frac{57}{275}-0.64 x-2 \max _{s \in[t-0.1, t]} x(s) \quad \text { for } t \in[0,0.35] \\
x(t)=-k_{1} \frac{3}{22}, \quad t \in[-0.1,0] .
\end{gathered}
$$

Choose $k_{1}=19 / 33$. Then the IVP (6.6) has an exact solution $\alpha_{2}(t)=-57 / 725$. It is clear that $\alpha_{0}(t) \leq \alpha_{1}(t) \leq \alpha_{2}(t)<0=x(t)$.

The first upper approximation $\beta_{1}(t)$ is a solution of the IVP

$$
\begin{gathered}
x^{\prime}(t)=\frac{37}{75}-0.64 x-2 \max _{s \in[t-0.1, t]} x(s) \quad \text { for } t \in[0,0.35] \\
x(t)=\frac{p_{0}}{4}, \quad t \in[-0.1,0] .
\end{gathered}
$$

Choose $p_{0}=74 / 99$. Then the IVP (6.7) has an exact solution $\beta_{1}(t)=37 / 198 \approx 0.1869$.

The second upper approximation $\beta_{2}(t)$ is a solution of the IVP

$$
\begin{gathered}
x^{\prime}=-\frac{114481}{398475}-0.64 x-2 \max _{s \in[t-0.1, t]} x(s) \quad \text { for } t \in[0,0.35], \\
x(t)=p_{1} \frac{37}{198}, \quad t \in[-0.1,0] .
\end{gathered}
$$

Choose $p_{1}=114481 / 196581$. Then the IVP (6.8) has an exact solution $\beta_{2}(t)=114481 /$ $1051974 \approx 0.108825$.

It is obviouse that $\beta_{0}(t)>\beta_{1}(t)>\beta_{2}(t)>0=x(t)>\alpha_{2}(t)>\alpha_{1}(t)>\alpha_{0}(t)$ on the interval $[-0.1,0.35]$.

Example 6.1 illustrates that the presence of the constants $p_{n}$ and $k_{n}$ allow us easily to construct each successive approximation in an explicit form.

Now we will illustrate the suggested method in the case when lower and upper solutions are defined for different initial points and on different intervals. 
Example 6.2. Consider the following IVP for the scalar nonlinear differential equation with "maxima":

$$
\begin{gathered}
x^{\prime}=\frac{t}{1+x(t)}-0.5 \max _{s \in[t-0.1, t]} x(s)-2 \text { for } t \in[1.5,1.75], \\
x(t)=0, \quad t \in[1.4,1.5], \\
x^{\prime}=\frac{t}{1+x(t)}-0.5 \max _{s \in[t-0.1, t]} x(s)-2 \text { for } t \in[2.35,2.6], \\
x(t)=0, \quad t \in[2.25,2.35],
\end{gathered}
$$

where $x \in \mathbb{R}$.

In this case, $f(t, x, y) \equiv t /(1+x)-0.5 y-2, t_{0}=1.5, \tau_{0}=2.35, \eta=\tau_{0}-t_{0}=0.85, T=0.25$, $r=0.1, J_{1}=[1.4,1.75], J_{2}=[2.25,2.6]$.

Choose $\alpha_{0}(t)=-1 / 4$. Then for $t \in[1.5,1.75]$ the inequality

$$
f\left(t, \alpha_{0}(t), \max _{s \in[t-0.1, t]} \alpha_{0}(s)\right)=\frac{t}{1-1 / 4}+\frac{1}{8}-2=\frac{4 t}{3}-\frac{15}{8} \geq 1.5 \frac{4}{3}-\frac{15}{8}=0.125>0
$$

holds. Therefore, $\alpha_{0}(t)$ is a lower solution of the IVP $(6.9)$ in $[1.4,1.75]$.

Let $\beta_{0}(t)=1 / 4$. Then for $t \in[2.35,2.6]$ the inequality

$$
f\left(t, \beta_{0}(t), \max _{s \in[t-0.1, t]} \beta_{0}(s)\right)=\frac{t}{1+1 / 4}-\frac{1}{8}-2=\frac{4 t}{5}-\frac{17}{8} \leq 2.6 \frac{4}{5}-\frac{17}{8}=-0.045<0
$$

holds. Therefore, $\beta_{0}(t)$ is an upper solution of the IVP $(6.10)$ on $[2.25,2.6]$.

In this case, $\tau_{0}>t_{0}$ and $\Omega\left(\alpha_{0}, \beta_{0}, \tau_{0}\right)=\left\{(t, u, v) \in[1.5,1.75] \times \mathbb{R}^{2}:-1 / 4 \leq u, v \leq 1 / 4\right\}$.

Let $t \in[1.5,1.76],-1 / 4 \leq u_{1} \leq u_{2} \leq 1 / 4$ and $-1 / 4 \leq v_{1} \leq v_{2} \leq 1 / 4$. Then from the inequality $t /\left(\left(1+u_{1}\right)\left(1+u_{2}\right)\right) \leq(16 / 9) t \leq 3.1111<3.2$, we obtain

$$
\begin{aligned}
f\left(t, u_{1}, v_{1}\right)-f\left(t, u_{2}, v_{2}\right) & =\frac{t}{1+u_{1}}-\frac{t}{1+u_{2}}+0.5\left(v_{2}-v_{1}\right) \\
& =\frac{t}{\left(1+u_{1}\right)\left(1+u_{2}\right)}\left(u_{2}-u_{1}\right)+0.5\left(v_{2}-v_{1}\right) \leq 3.2\left(u_{2}-u_{1}\right)+\left(v_{2}-v_{1}\right)
\end{aligned}
$$

That is, $L_{1}=3.2, L_{2}=0.5$, and the inequality $\left(L_{1}+L_{2}\right) T<1$ holds.

According to Theorem 4.1, there exist two convergent monotone sequences of functions $\left\{\alpha_{n}(t)\right\}_{0}^{\infty}$ and $\left\{\beta_{n}(t)\right\}_{0}^{\infty}$ such that (4.3) holds and their limits are solutions of the IVP (6.9) and (6.10). 


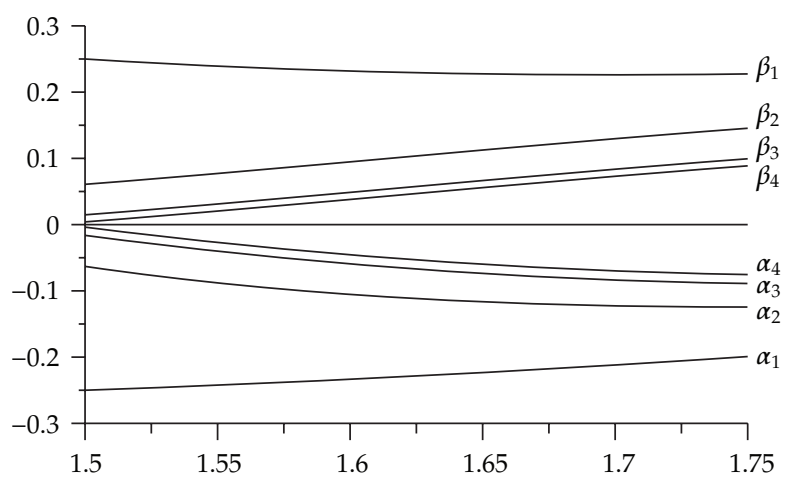

Figure 1: (graphs of $\alpha_{k}(t)$ and $\left.\beta_{k}(t+.85), k=1,2,3,4\right)$.

The successive approximations $\alpha_{n+1}(t)$ will be the unique solutions of the linear problems

$$
\begin{aligned}
x^{\prime} & =\frac{t}{1+\alpha_{n}(t)}-0.5 \alpha_{n}(t)-2-3.2\left(x-\alpha_{n}(t)\right)-0.5\left(\max _{s \in[t-0.1, t]} x(s)-\max _{s \in[t-0.1, t]} \alpha_{n}(s)\right) \\
& =\frac{t}{1+\alpha_{n}(t)}+2.7 \alpha_{n}(t)+0.5 \max _{s \in[t-0.1, t]} \alpha_{n}(s)-2-3.2 x-0.5 \max _{s \in[t-0.1, t]} x(s) \quad \text { for } t \in[1.5,1.75], \\
x(t) & =-k_{n} L_{n} \quad \text { for } t \in[1.4,1.5],
\end{aligned}
$$

and the successive approximations $\beta_{n+1}(t)$ will be the unique solutions of the linear problems

$$
\begin{aligned}
x^{\prime}(t) & =\frac{t}{1+\beta_{n}(t)}+2.7 \beta_{n}(t)+0.5 \max _{s \in[t-0.1, t]} \beta_{n}(s)-2-3.2 x-0.5 \max _{s \in[t-0.1, t]} x(s) \quad \text { for } t \in[2.35,2.6], \\
x(t) & =p_{n} C_{n} \text { for } t \in[2.25,2.35]
\end{aligned}
$$

where $\alpha_{0}(t)=-1 / 4, \beta_{0}(t)=1 / 4, L_{n}=C_{n}=0.25, k_{n}=0.25^{n}, p_{n}=0.25^{n}$, and $n=1,2,3, \ldots$

Note the above IVPs are linear, but we are not able to obtain their solutions in explicit form because of the presence of maximum function.

We will use the computer realization of the considered method, explained in Section 4, to solve the initial value problems (6.14) and (6.15) for $n=1,2,3,4,5$. In this case at any step, the function $y_{n}\left(t_{k}\right)$ in (5.1) is replaced by the functions $\alpha_{n}\left(t_{k}\right)$ or $\beta_{n}\left(t_{k}\right)$, respectively, the function $y_{n+1}\left(t_{k}\right)$ is the next approximation $\alpha_{n+1}\left(t_{k}\right)$ or $\beta_{n+1}\left(t_{k}\right)$, respectively, and the function $f$ is the right part of (6.14) or (6.15), respectively, and $h=0.00001$. Some of the numerical values of the successive approximations $\left\{\alpha_{n}(t)\right\}_{0}^{\infty}$ and $\left\{\beta_{n}(t)\right\}_{0}^{\infty}$, which are lower/upper solutions of the given problem, are shown in the Table 1. Also, the obtained successive approximations are graped on the Figure 1. Both, Table 1 and Figure 1, illustrate us the monotonicity of the sequences $\left\{\alpha_{n}(t)\right\}_{0}^{\infty}$ and $\left\{\beta_{n}(t)\right\}_{0}^{\infty}$ and the validity of the inequalities

$$
\alpha_{0}(t) \leq \alpha_{1}(t) \leq \cdots \leq \alpha_{n}(t) \leq \beta_{n}(t+.85) \leq \cdots \leq \beta_{0}(t+.85), \quad t \in[1.5,1.75] .
$$


Table 1: Values of the successive lower/upper approximations $\alpha_{k}(t)$ and $\beta_{k}(t+.85)$.

\begin{tabular}{lcccccc}
\hline$t$ & 1.50 & 1.55 & 1.60 & 1.65 & 1.70 & 1.75 \\
\hline$\beta_{1}(t+.85)$ & 0.2500000 & 0.2396285 & 0.2326388 & 0.2286608 & 0.2273177 & 0.2281468 \\
$\beta_{2}(t+.85)$ & 0.0625000 & 0.0795487 & 0.0954878 & 0.1138875 & 0.1307175 & 0.1464314 \\
$\beta_{3}(t+.85)$ & 0.0156250 & 0.0322634 & 0.0490684 & 0.0671041 & 0.0850914 & 0.1029515 \\
$\beta_{4}(t+.85)$ & 0.0039063 & 0.0211106 & 0.0383552 & 0.0558882 & 0.0734248 & 0.0909491 \\
$\beta_{5}(t+.85)$ & 0.0009766 & 0.0183982 & 0.0358232 & 0.0532996 & 0.0707523 & 0.0881732 \\
\hline$\alpha_{5}(t)$ & -0.0009766 & -0.0236676 & -0.0416446 & -0.0552493 & -0.0648509 & -0.0708277 \\
$\alpha_{4}(t)$ & -0.0039063 & -0.0269991 & -0.0452915 & -0.0591247 & -0.0688717 & -0.0749110 \\
$\alpha_{3}(t)$ & -0.0156250 & -0.0399773 & -0.0590208 & -0.0731539 & -0.0828101 & -0.0884511 \\
$\alpha_{2}(t)$ & -0.0625000 & -0.0871172 & -0.1048556 & -0.1164338 & -0.1225992 & -0.1243204 \\
$\alpha_{1}(t)$ & -0.2500000 & -0.2427252 & -0.2336358 & -0.2230384 & -0.2111877 & -0.1982954 \\
\hline
\end{tabular}

\section{Acknowledgments}

This paper was partially supported by Fund "Scientific Research" NI11FMI004/30.05.2011, Plovdiv University and BG051PO001/3.3-05-001 Science and Business, financed by the Operative Program “Development of Human Resources," European Social Fund.

\section{References}

[1] E. P. Popov, Automatic Regulation and Control, NASA, Moscow, Russia, 1966.

[2] R. P. Agarwal, D. R. Anderson, and A. Zafer, "Interval oscillation criteria for second-order forced delay dynamic equations with mixed nonlinearities," Computers and Mathematics with Applications, vol. 59, no. 2, pp. 977-993, 2010.

[3] M. U. Akhmetov and A. Zafer, "Successive approximation method for quasilinear impulsive differential equations with control," Applied Mathematics Letters, vol. 13, no. 5, pp. 99-105, 2000.

[4] J. Bastinec, L. Berezansky, J. Diblik, and Z. Smarda, "On the critical case in oscillation for differential equations with a single delay and with several delays," Abstract and Applied Analysis, vol. 2010, Article ID 417869, 20 pages, 2010.

[5] L. Berezansky and E. Braverman, "On stability of some linear and nonlinear delay differential equations," Journal of Mathematical Analysis and Applications, vol. 314, no. 2, pp. 391-411, 2006.

[6] L. Berezansky and E. Braverman, "Positive solutions for a scalar differential equation with several delays," Applied Mathematics Letters, vol. 21, no. 6, pp. 636-640, 2008.

[7] L. Berezansky and E. Braverman, "New stability conditions for linear differential equations with several delays," Abstract and Applied Analysis, vol. 2011, Article ID 178568, 19 pages, 2011.

[8] J. Diblik, "Asymptotic convergence criteria of solutions of delayed functional differential equations," Journal of Mathematical Analysis and Applications, vol. 274, no. 1, pp. 349-373, 2002.

[9] J. Diblik and N. Koksch, "Existence of global solutions of delayed differential equations via retract approach," Nonlinear Analysis, vol. 64, no. 6, pp. 1153-1170, 2006.

[10] J. Diblik and M. Kudelcikova, "Existence and asymptotic behavior of positive solutions of functional differential equations of delayed type," Abstract and Applied Analysis, vol. 2011, Article ID 754701, 16 pages, 2011.

[11] J. Diblik and M. Ruzickova, "Convergence of the solutions of the equation $\dot{y}(t)=\beta(t)[y(t-\delta)-y(t-\tau)]$ in the critical case," Journal of Mathematical Analysis and Applications, vol. 331, no. 2, pp. 1361-1370, 2007.

[12] J. Diblik, M. Ruzickova, and Z. Suta, "Asymptotical convergence of the solutions of a linear differential equation with delays," Advances in Difference Equations, vol. 2010, Article ID 749852, 12 pages, 2010.

[13] D. Franco, J. J. Nieto, and Y. V. Rogovchenko, "Periodic boundary value problem for nonlinear first order ordinary differential equations with impulses at fixed moments," Extracta Mathematicae, vol. 13, no. 3, pp. 313-326, 1998. 
[14] I. Gyori and F. Hartung, "Stability in delay perturbed differential and difference equations," Fields Institute Communications, vol. 29, pp. 181-194, 2001.

[15] D. Bainov and S. Hristova, Differential Equations with Maxima, Chapman and Hall/CRC, 2011.

[16] D. D. Bainov and S. G. Hristova, "Monotone-iterative techniques of Lakshmikantham for a boundary value problem for systems of differential equations with 'maxima'," Journal of Mathematical Analysis and Applications, vol. 190, no. 2, pp. 391-401, 1995.

[17] S. W. Du and V. Lakshmikantham, "Monotone iterative technique for differential equations in a Banach space," Journal of Mathematical Analysis and Applications, vol. 87, no. 2, pp. 454-459, 1982.

[18] S. Heikkila and V. Lakshmikantham, Monotone Iterative Techniques for Discontinuous Nonlinear Differential Equations, vol. 181 of Monographs and Textbooks in Pure and Applied Mathematics, Marcel Dekker, New York, NY, USA, 1994.

[19] V. Lakshmikantham and A. S. Vatsala, "General uniqueness and monotone iterative technique for fractional differential equations," Applied Mathematics Letters, vol. 21, no. 8, pp. 828-834, 2008.

[20] J. J. Nieto, Y. Jiang, and Y. Jurang, "Monotone iterative method for functional-differential equations," Nonlinear Analysis, vol. 32, no. 6, pp. 741-747, 1998.

[21] P. Y. H. Pang and R. P. Agarwal, "Monotone iterative methods for a general class of discrete boundary value problems," Computers and Mathematics with Applications, vol. 28, no. 1-3, pp. 243-254, 1994.

[22] T. Jankowski, "Differential inequalities with initial time difference," Applicable Analysis, vol. 81, no. 3, pp. 627-635, 2002.

[23] T. Jankowski, "Delay integro-differential inequalities with initial time difference and applications," Journal of Mathematical Analysis and Applications, vol. 291, no. 2, pp. 605-624, 2004.

[24] A. Li, W. Lv, and J. Ye, "Exponential and global stability of nonlinear dynamical systems relative to initial time difference," Applied Mathematics and Computation, vol. 217, no. 12, pp. 5923-5929, 2011.

[25] A. Li, E. Feng, and S. Li, "Stability and boundedness criteria for nonlinear differential systems relative to initial time difference and applications," Nonlinear Analysis. Real World Applications, vol. 10, no. 2, pp. 1073-1080, 2009.

[26] V. Lakshmikantham, S. Leela, and J. Vasundhara, "Another approach to the theory of differential inequalities relative to changes in the initial times," Journal of Inequalities and Applications, vol. 4, no. 2, pp. 163-174, 1999.

[27] V. Lakshmikantham and A. S. Vatsala, "Differential inequalities with initial time difference and applications," Journal of Inequalities and Applications, vol. 3, no. 3, pp. 233-244, 1999.

[28] Y. Zhang and Z. Yi, "Theory of functional differential equation with initial data difference," Dynamic Systems and Applications, vol. 10, no. 4, pp. 523-532, 2001.

[29] C. Yakar and M. D. Shaw, "A comparison result and Lyapunov stability criteria with initial time difference," Dynamics of Continuous, Discrete and Impulsive Systems Series A, vol. 12, no. 6, pp. 731-737, 2005.

[30] C. Yakar and M. Shaw, "Practical stability in terms of two measures with initial time difference," Nonlinear Analysis, vol. 71, no. 12, pp. 781-785, 2009.

[31] B. Ahmad, "Integro-differential equations with initial time difference," Dynamic Systems and Applications, vol. 16, no. 3, pp. 497-506, 2007.

[32] L. Skora, "Impulsive differential equations with initial data difference," Commentationes Mathematicae, vol. 51, no. 1, pp. 55-69, 2011.

[33] C. Yakar and A. Yakar, "Monotone iterative technique with initial time difference for fractional differential equations," Hacettepe Journal of Mathematics and Statistics, vol. 40, no. 2, pp. 331-340, 2011.

[34] A. Cabada and J. J. Nieto, "Rapid convergence of the iterative technique for first order initial value problems," Applied Mathematics and Computation, vol. 87, no. 2-3, pp. 217-226, 1997.

[35] I. Gyori, "Global attractivity in delay differential equations using a mixed monotone technique," Journal of Mathematical Analysis and Applications, vol. 152, no. 1, pp. 131-155, 1990.

[36] I. Gyori and J. Turi, “Uniform approximation of a nonlinear delay equation on infinite intervals," Nonlinear Analysis, vol. 17, no. 1, pp. 21-29, 1991.

[37] S. G. Hristova and D. D. Bainov, "Application of the monotone-iterative techniques of V. Lakshmikantham for solving the initial value problem for impulsive differential-difference equations," The Rocky Mountain Journal of Mathematics, vol. 23, no. 2, pp. 609-618, 1993.

[38] S. G. Hristova and D. D. Bainov, "Application of the monotone-iterative techniques of V. Lakshmikantham to the solution of the initial value problem for impulsive differential equations with 'supremum'," Journal of Mathematical and Physical Sciences, vol. 25, no. 1, pp. 69-80, 1991.

[39] G. S. Ladde, V. Lakshmikantham, and A. S. Vatsala, Monotone Iterative Techniques for Nonlinear Differential Equations, Pitman, New York, NY, USA, 1985. 
[40] V. Lakshmikantham and B. G. Zhang, "Monotone iterative technique for delay differential equations," Applicable Analysis, vol. 22, no. 3-4, pp. 227-233, 1986.

[41] Y. Xing, M. Han, and G. Zheng, "Initial value problem for first-order integro-differential equation of Volterra type on time scales," Nonlinear Analysis, vol. 60, no. 3, pp. 429-442, 2005.

[42] Sh. Zhang, "Monotone iterative method for initial value problem involving Riemann-Liouville fractional derivatives," Nonlinear Analysis, vol. 71, no. 5-6, pp. 2087-2093, 2009.

[43] A. Golev, S. Hristova, and A. Rahnev, "An algorithm for approximate solving of differential equations with 'maxima'," Computers and Mathematics with Applications, vol. 60, no. 10, pp. 2771-2778, 2010.

[44] V. G. Angelov and D. D. Bainov, "On the functional-differential equations with 'maxima'," Applicable Analysis, vol. 16, no. 3, pp. 187-194, 1983. 


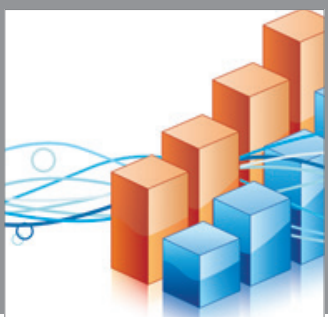

Advances in

Operations Research

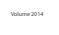

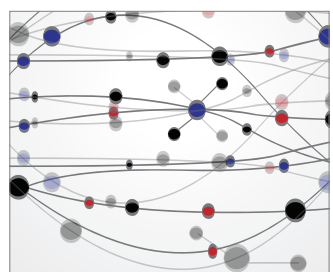

\section{The Scientific} World Journal
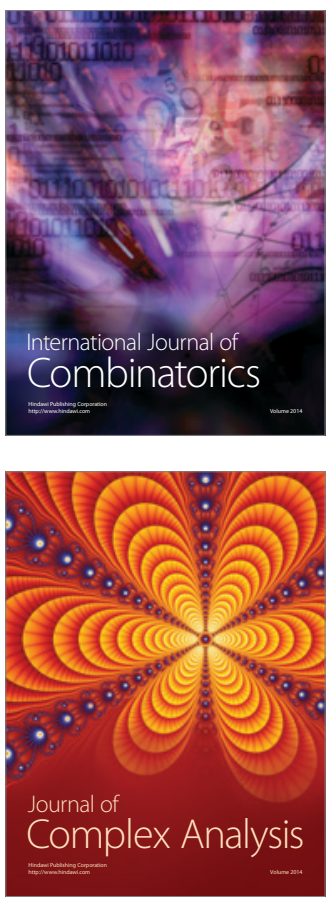

International Journal of

Mathematics and

Mathematical

Sciences
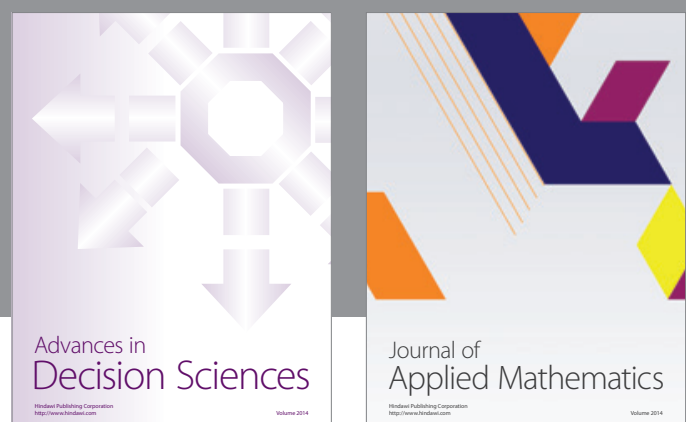

Journal of

Applied Mathematics
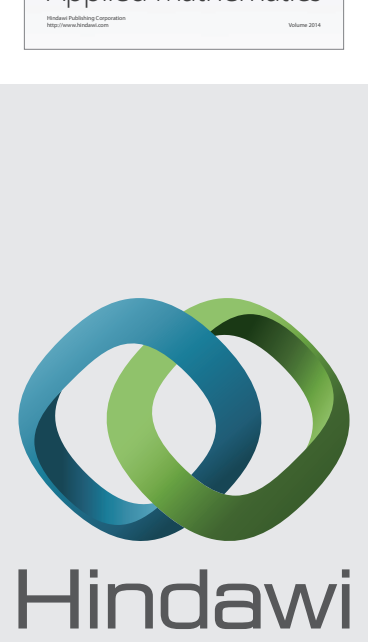

Submit your manuscripts at http://www.hindawi.com
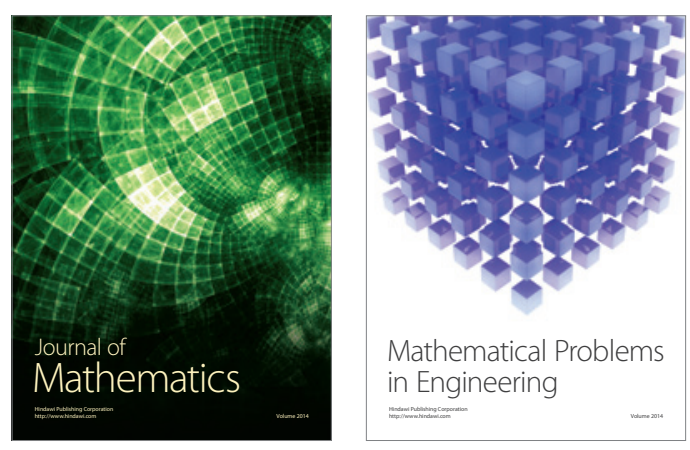

Mathematical Problems in Engineering
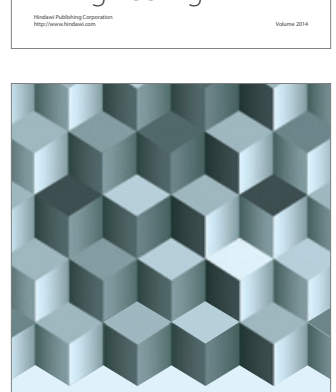

Journal of

Function Spaces
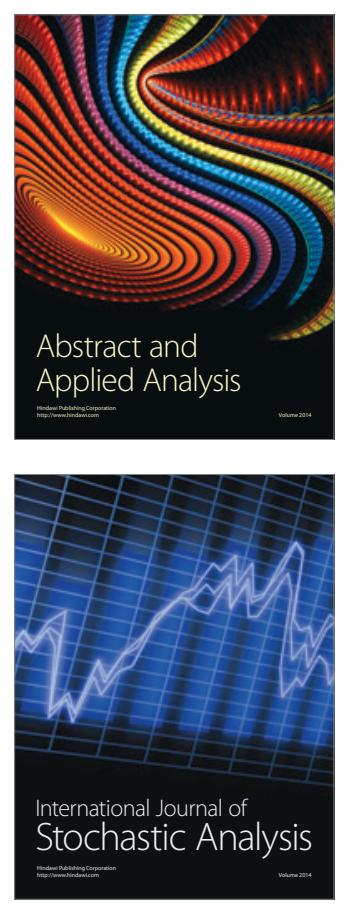

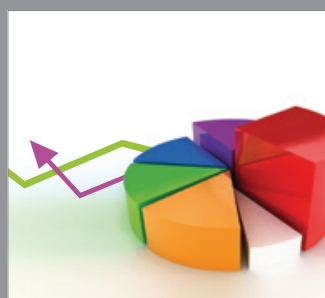

ournal of

Probability and Statistics

Promensencen
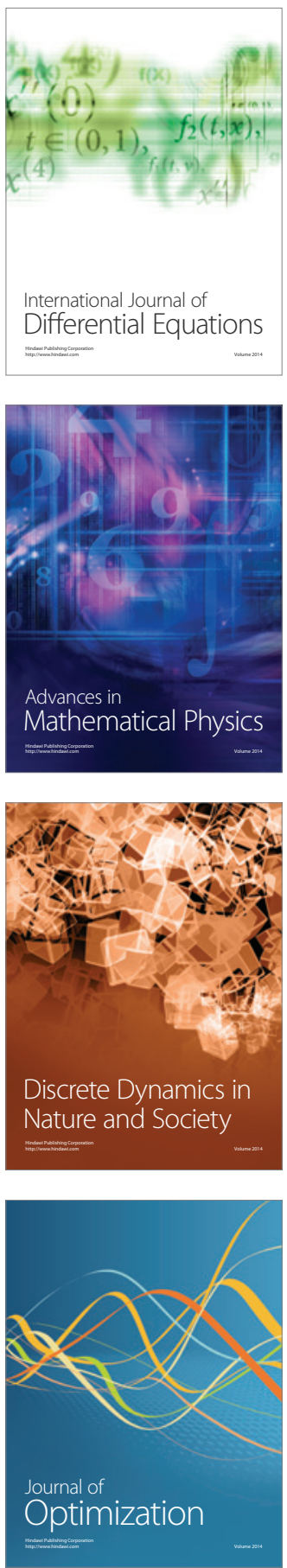